\title{
Antibody Engineering - Tailor-Made Next Generation Antibodies by Molecular Design
}

\author{
Andreas Humpe ${ }^{a}$ Matthias Peipp ${ }^{b}$ \\ a Institute of Transfusion Medicine and Immune Hematology with Blood Bank, University Hospital Magdeburg. Magdeburg, Germany; \\ ${ }^{b}$ Division of Stem Cell Transplantation and Immunotherapy, Department of Medicine II, Christian-Albrechts-University Kiel, Kiel, Germany
}

The implementation of therapeutic antibodies has revolutionized the therapy of cancer patients. Based on the clinical success of prototypic tumor-targeting antibodies like rituximab and trastuzumab, the way for a variety of next-generation antibodies with improved characteristics was opened. Innovations in techniques to generate humanized or even human molecules (e.g. phage display, mice engineered to express human antibody repertoires) were key for the clinical triumph of first-generation antibodies. Despite the proven clinical activity in different cancer entities, not all patients respond to antibody therapy. Consequently, novel approaches to improve this generally well tolerated therapy are major areas of research in academia and in pharmaceutical industry. A deeper understanding of pathophysiological coherences, identification of critical checkpoints in the interaction of tumor cells and the immune system as well as novel insights into the mechanisms of action mediated by therapeutic antibodies have fueled innovations in antibody engineering. Obvious engineering strategies include approaches in improving IgG intrinsic effector mechanisms such as antibody-dependent cell-mediated cytotoxicity (ADCC), antibodydependent cellular phagocytosis (ADCP), or complement-dependent cytotoxicity (CDC). Besides engineering conventional IgG antibodies, innovative antibody derivatives such as bispecific antibodies, antibody drug conjugates, and antibody fusion proteins have been developed and corresponding prototypic agents were recently approved or are in various phases of clinical development. Several aspects of established and emerging antibody engineering technologies are addressed in this issue of Transfusion Medicine ANd Hemotherapy (fig. 1).

Frenzel and colleagues [1] summarize opportunities and achievements of phage display, including the generation of antibodies with specificities which could not be generated otherwise. To date, the IgG1 isotype is predominantly used in antibody therapy. Kretschmer et al. [2] review the potential of alternative human isotypes in therapeutic application. Kellner et al. [3] summarize established and emerging Fc engineering strategies, whereas Wirt

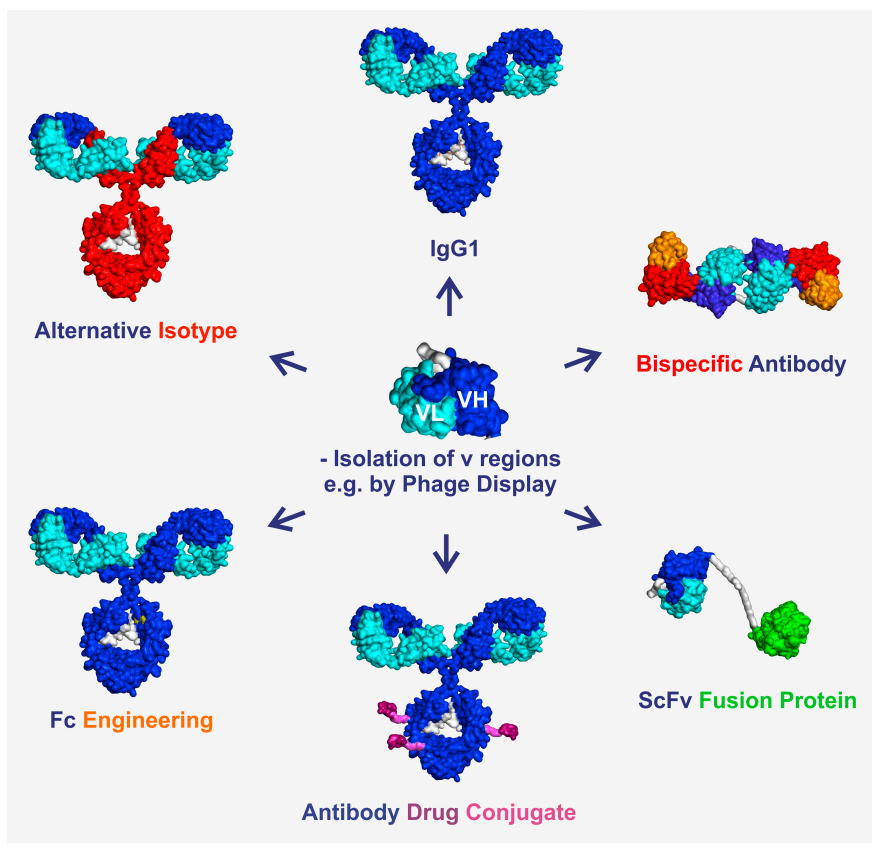

Fig. 1. Graphical overview of antibody engineering strategies addressed in this issue. Based on carefully selected antibody $\mathrm{v}$ regions a variety of antibody engineering techniques are available to design fit-for-purpose antibody molecules with tailor-made effector functions for a given clinical application. IgG model structures are based on an IgG1 pdb file kindly provided by Dr. M. Clark. The Fc engineered antibody displays two amino acid exchanges for improved Fc $\gamma$ RIIa and Fc $\gamma$ RIIIa binding. The antibody drug conjugate model represents an IgG1 maytansine conjugate; the bispecific antibody model illustrates the prototype TandAb moleacule AFM13. AFM13 is bivalent for Fc $\gamma$ RIIIA (red/orange) on NK cells and bivalent for the tumor antigen CD30 (blue).The $\mathrm{scFv}$-fusion protein shows a homology model of a scFv fragment fused to the so-called SNAP-Tag (green). Model structures are generated / illustrated using YASARA and Discovery Studio Visualizer software packages.

and colleagues [4] analyze the potential of combining different Fc engineering strategies. Koch and Tesar [5] review the concept of bispecific antibodies as effector cell engagers and discuss promis-

\section{KARGER}

() 2017 S. Karger GmbH, Freiburg 
ing settings for optimal clinical application. Finally, Padayachee and colleagues [6] discuss the potential of antibody drug conjugates and corresponding recombinant antibody fusion proteins in the background of breast cancer.
Together the set of articles gives a brief overview in currently established and emerging antibody engineering strategies to improve immunotherapy of cancer.

\section{References}

1 Frenzel A, Kügler J, Helmsing S, Meier D, Schirrmann T, Hust M, Dübel S: Designing human antibodies by phage display. Transfus Med Hemother 2017;44: DOI $10.1159 / 000479633$.

2 Kretschmer A, Schwanbeck R, Valerius T, Rösner T: Antibody isotypes for tumor immunotherapy. Transfus Med Hemother 2017;44: DOI: 10.1159/000479240.

3 Kellner C, Otte A, Cappuzzello E, Klausz K, Peipp M: Modulating cytotoxic effector functions by Fc engineering to improve cancer therapy. Transfus Med Hemother 2017 44: DOI: $10.1159 / 000479980$
4 Wirt T, Rosskopf S, Rösner T, Eichholz K, Kahrs A, Lutz S, Kretschmer A, Valerius T, Klausz K, Otte A, Gramatzki M, Peipp M, Kellner C: An Fc double-engineered CD20 antibody with enhanced ability to trigger complement-dependent cytotoxicity and antibody-dependent cell-mediated cytotoxicity. Transfus Med Hemother 2017;44 DOI: $10.1159 / 000479978$.

5 Koch J, Tesar M: Recombinant antibodies to arm cytotoxic lymphocytes in cancer immunotherapy. Transfus Med Hemother 2017;44: DOI: 10.1159/000479981.

6 Padayachee E, Biteghe F, Malindi Z, Bauerschlag D, Barth S: Human antibody fusion proteins / antibody drug conjugates. Transfus Med Hemother 2017;44: DOI: $10.1159 / 000479979$. 